\title{
MENINGITE EOSINOFÍLICA E EOSINOFILIA SANGÜÍNEA DE ORIGEM PARASITÁRIA
}

\author{
EHRENFRIED O. WITTIG * \\ IZRAIL CAT ** \\ LeIDE P. MarinoNi ***
}

Quadros eosinofílicos sangüíneos são de observação freqüente, principalmente em paises tropicais. As parasitoses intestinais, especialmente as helmintíases, constituem a causa mais comum de eosinofilia sangüínea $8,25,35$.

Numerosos agentes podem determinar eosinofilia à qual, em algumas situações, se associam manifestações clínicas, constituindo quadros mais complexos, alguns dos quais já foram agrupados em sindromes ${ }^{3,37}$. A sindrome de Weingarten ${ }^{7,40,54}$ caracterizada por leucocitose, eosinofilia intensa, crises asmatiformes e hipertermia, tem evolução às vezes prolongada, sendo sua etiologia atribuída a parasitoses. Freqüentemente é de cura medicamentosa e incide habitualmente em países tropicais (eosinofilia tropical). Na sindrome de Löeffler ${ }^{15}$, caracterizada por manifestações subjetivas, eosinofilia moderada, infiltrado pulmonar transitório mais evidente que na síndrome de Weingarten, com evolução rápida e cura espontânea, a etiologia também é atribuida a parasitoses.

Embora sem a devida comprovação de infestação zoonótica no homem, a esse fator foram atribuídos numerosos casos de eosinofilia. Beaver e col. ${ }^{4}$ foram os primeiros a documentar a infestação por larvas no homem, identificando em seus dois casos iniciais, larvas de Toxocara canis. Beaver estabeleceu ainda que este processo é devido a infestação por ovos de parasitas, os quais libertam a forma larvar, que migra pelo organismo, determinando acentuada eosinofilia, hepatomegalia, esplenomegalia, febre recurrente, irritabilidade, hiperglobulinemia, alteraçōes oftalmológicas, com maior incidência em crianças. Este quadro Beaver denominou de "Larva migrans visceral".

Várias outras patologias podem apresentar eosinofilia sangüínea 19, 48 embora com manifestações menos exuberantes, merecendo serem mencionadas a pseudo-leucemia eosinofílica e a eosinofilia familiar ${ }^{12}$.

Além do sangue, outros humores ou tecidos podem apresentar reações eosinofílicas $2,17,23,28,53$. O sistema nervoso central e as meninges podem ser sede de reações eosinofílicas, exteriorizadas principalmente no líquido céfalorraqueano $21,22,24,32,33,37$. 
Os principais agentes responsáveis por esta forma de reação são a torulose ${ }^{38}$ e as parasitoses ${ }^{16,18}$, das quais destacamos a ascaridiase 20,45 , a esquistossomose ${ }^{11}, 44,51$, a cisticercose ${ }^{30}, 49,50$, a hidatidose ${ }^{42} \mathrm{e}$, ainda, as infestações por Angiostrongylus ${ }^{39}$ e Gnathostoma ${ }^{13}$.

$\mathrm{Na}$ América do Sul aproximadamente $2 / 3$ dos casos de eosinofilia liquórica são devidos a neurocisticercose. A meningencefalite eosinofílica pode ocorrer esporádicamente ou endêmicamente como já foi registrado na área do Pacífico. A grande maioria dos casos foi observada no Haiti, onde mais de duas centenas foram relatados ${ }^{10,41}$. Até 1961, haviam sido descritos casos isolados na França (4), na Suíça (3), na Espanha (1), na Alemanha (1), nos Estados Unidos da América do Norte (3), no Paraguai (1) e no Japão (1) ${ }^{46}$.

A meningite eosinofílica é eventualidade rara e a sua associação com intensa eosinofilia sangüínea, hepatomegalia e esplenomegalia, constitui quadro clínico não relatado em nosso país. Este registro de um caso de meningencefalite eosinofílica é o terceiro na América Latina ${ }^{14}{ }^{46}$ e o primeiro no Brasil, segundo a literatura consultada.

\section{O B S E R V C Ã O}

S.R.F., com 12 meses de idade, sexo masculino, branco, natural e residente em Joinvile, Estado de Santa Catarina, internado em 02-03-1967, no Pronto Socorro Infantil São Luiz (R.G. 1086). História clinica - Doença iniciada 45 dias antes da internação com hipertermia diária, contínua durante 15 dias, apresentando por 4 vezes crises convulsivas tônicas, com desvio ocular para cima e duração de 15 minutos, com eliminação de Ascaris lumbricoides por via oral. Antecedentes - Parto e gestação normais. Infecçōes de vias aéreas superiores e enterocolopatias. Três dias antes do inicio do quadro atual a criança foi mordida por cachorro na região malar esquerda, sem solução de continuidade na pele. Exame físico - Peso $10.600 \mathrm{~g}$; temperatura $37,5^{\circ} \mathrm{C}$; figado $4 \mathrm{~cm}$ abaixo do rebordo costal, na linha hemiclavicular direita, indolor, bordo rombo, superfície lissa, consistência um pouco aumentada; baço a $2 \mathrm{~cm}$ do rebordo costal na linha hemiclavicular esquerda. Exame neurológico $\mathrm{Na}$ fundoscopia ocular as veias mostravam-se moderadamente ingurgitadas, com aumento de tortuosidades, retina brilhante, papila com bordos nitidos e miopia. Discreta rigidez de nuca. Irritabilidade. Exames complementares - Eletrencefalograma normal. Exame parasitológico: Ascaris lumbricoides. Reacão de Weinberg no sangue negativa. Exame de urina normal. Hemograma: hemácias 3.660.000; hemoglobina $9,65 \%$; leucócitos $54.500 \mathrm{~mm}^{3}$ (eosinófilos $72 \%$; linfócitos $20 \%$; monócitos $3 \%$; neutrófilos $5 \%$ ). Liquido céfalorraqueano: discretamente xantocrônico; células $187 / \mathrm{mm}^{3}$ (linfócitos $14 \%$; basófilos $3 \%$; reticulócitos $5 \%$; eosinófilos $78 \%$ ); glicose $42 \mathrm{mg} \%$; proteínas $70 \mathrm{mg} \%$; reações para globulinas levemente positivas; R. Wassermann negativa; R. Weinberg negativa; bacterioscopia negativa; pesquisa para fungos negativa; cultura em agar-sangue negativa. O exame de líquido céfalorraqueano e o hemograma foram repetidos para confirmação dos resultados, tendo sido novamente encontrados eosinófilos em porcentagem elevada (78\% e $75 \%$, respectivamente). Biópsia hepática: "Processo inflamatório granulomatoso eosinofílico" (Fig. 1 e 2).

O paciente foi medicado com penicilina e piperazina, com o que eliminou grande quantidade de Ascaris lumbricoides, havendo regressão rápida e parcial da hepatoesplenomegalia. A criança teve alta após 14 dias em melhores condições. Nova revisão somente foi feita em 1971 quando foi verificado que persistiam apenas as crises convulsivas, sendo o desenvolvimento psicomotor aparentemente normal. Hemograma (1971): hemácias 4.480.000 $\mathrm{mm}^{3}$; hemoglobina 13,3 $\mathrm{g} \%$; leucócitos $12.100 \mathrm{~mm}^{3}$ (eosinófilos $13 \%$; linfócitos $31 \%$; monócitos $7 \%$; neutrófilos $49 \%$ ); plaquetas normais. 

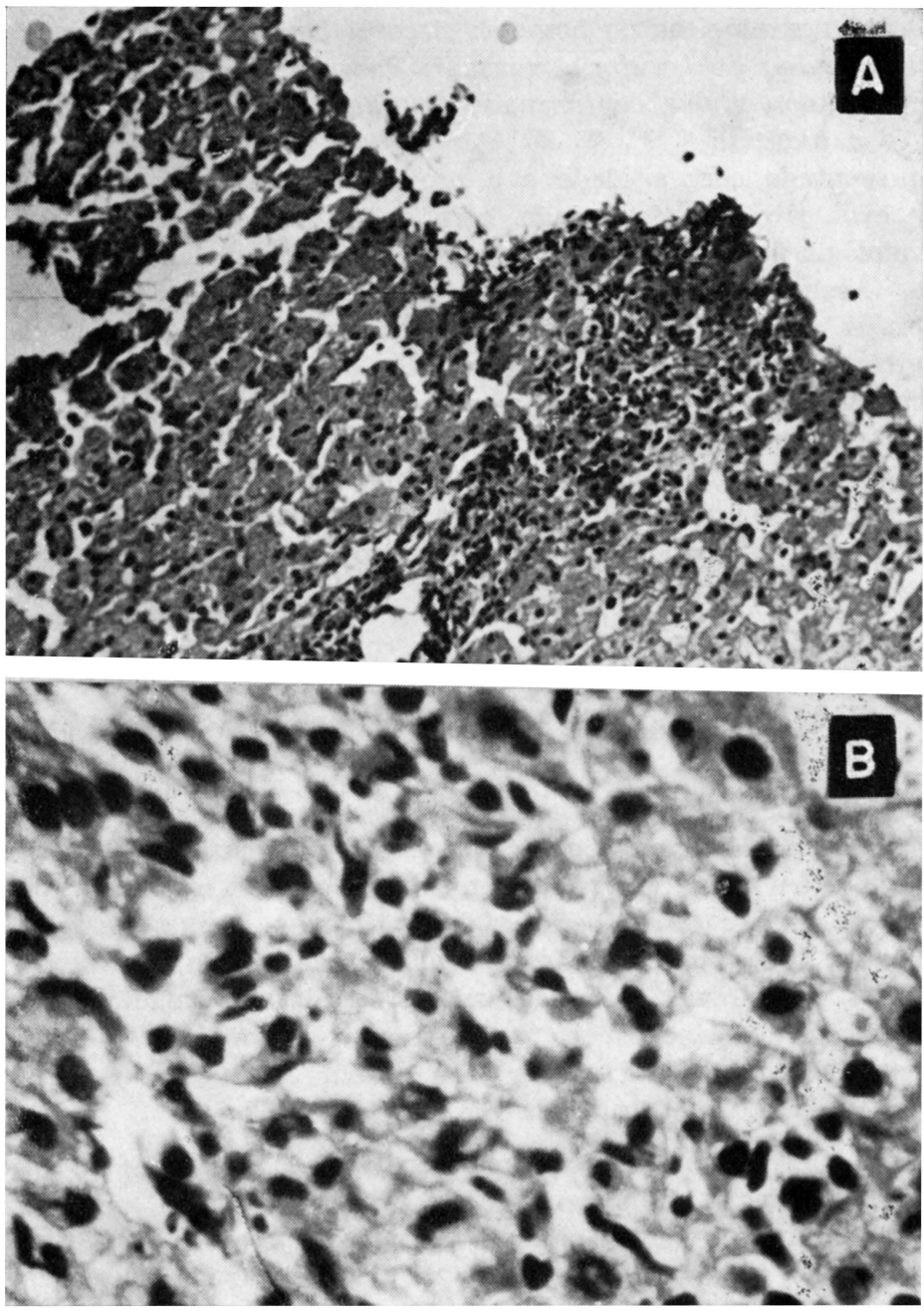

Fig. 1 - Caso S.R.F. Meningite eosinofilica. Biópsia hepática: em $A$, espaço portal com reação inflamatória granulomatosa (HE 170x); em $B$, reação inflamatória com células mononucleadas e abundantes leucócitos eosinófilos (HE 400x).

\section{COMENTARIOS}

A denominação de meningite eosinofílica é sugerida para os casos em que a taxa de eosinofilorraquia é superior a $30 \%{ }^{45}$. A etiologia deste tipo de meningite, embora ainda pouco esclarecida para a maioria dos autores é freqüentemente atribuída à infestação parasitária e, em especial, às parasitoses helminticas. Os seguintes parasitas já foram responsabilizados por 
manifestações neurológicas no homem: Ascaris lumbricoides; Toxocara canis; Trichinella spiralis; Schistosoma mansoni; Paragonimus westermani; Fasciola hepatica; Angiostrongylus cantonensis; Gnathostoma spinigerum; e, ainda, o cistiterco e a hidatide $6,20,26,27,29,31,34,38,47$. Relacionando o quadro clínico apresentado com a idade e a procedência do nosso paciente, com o tempo de evolução da enfermidade, com os resultados dos exames de fezes e a epidemiologia parasitária podemos estabelecer, por exclusão, seu diagnóstico etiológico. Assim: a) a infestação do sistema nervoso central pela Trichinella spirallis produz apenas moderada reação eosinofílica; $b$ ) não foram ainda registrados na cidade de Joinvile casos de esquistossomose; $c$ ) não há relatos no Brasil de infestações humanas, por Fasciola e Paragonimus e, nesstes casos, a sintomatologia é diferente, não havendo também referências à existência de Angiostrongylus e Gnathostoma em nosso meio; d) a cisticercose não produz hepatoesplenomegalia ou eosinofilia tão acentuada; $e$ ) a hidatidose, determina moderada eosinofilia no líquido céfalorraqueano sem chegar à meningite eosinofílica.

Conseqüentemente, das parasitoses já conhecidas como agentes de processos neuropatológicos no homem, permanecem como possibilidades etiológicas, as manifestações devidas às larvas de Ascaris lumbricoides ou de Toxocara canis.

O quadro clínico nestas duas infestações pode diferir apreciavelmente. O Ascaris lumbricoides, no seu ciclo hepato-pulmonar habitual no homem, principalmente em crianças, pode determinar cefaléia, vômitos, convulsões, febre, agitação, rush cutâneo, hemorragias retinianas, edema palpebral, hepatoesplenomegalia, bronquite, pneumonia clínica e radiológica e aumento de taxa de eosinófilos no sangue e líquido céfalorraqueano. Entretanto a hepatoesplenomegalia e a eosinofilia no sangue e no líquido céfalorraqueano verificados neste caso foram excessivas para serem atribuídas à ascaridose de acordo com a literatura consultada 6, 20 .

Por outro lado a infestação humana pelo Toxocara canis determina sintomatologia exuberante (fenômeno de impasse). A Larva migrans visceral, mais comum em crianças de 6 meses a 4 anos, apresenta lesões granulomatosas eosinofílicas no fígado e às vezes na pele, infiltrado pulmonar, hepatoesplenomegalia, febre intermitente, inapetência, dores musculares, articulares e abdominais. Podem ocorrer também erupções cutâneas ou edema de Quincke, linfoadenopatias, lesões oculares, granulomas subcutâneos, prurido, leucocitose, eosinofilia intensa e hiperglobulinemia. A evolução para a cura é freqüentemente espontânea e rápida. Algumas vezes o sistema nervoso é comprometido, tendo sido este fato documentado anátomo-patologicamente ${ }^{36,52}$.

O achado de larva parasitária principalmente em biópsias, depende da fase ou tempo de infestação, assim como do local, sendo um destes provavelmente o motivo de sua ausência na biópsia hepática por nós realizada $1,5,9$, $36,43,47,54,55$.

Durante a internação, nosso paciente foi medicado com penicilina mas, levando-se em consideração as hipóteses etiológicas aventadas, esta medicação não deve ter influido na melhora. No caso em estudo, o quadro clínico de 
febre persistente, convulsões, hepatoesplenomegalia, rigidez de nuca, acentuada eosinofilia no sangue e no líquido céfalorraqueano, com regressão espontânea, parece indicar o Toxocara canis como agente etiológico.

Parece pouco provável a possibilidade de causas não parasitárias terem influido: a medicamentosa, por falta de qualquer referência a tratamento anterior; a alérgica (exceto a reação ao parasita ou material do seu catabolismo ou destruição, ovos ou larvas) que nãc se acompanha de hepatoesplenomegalia, nem de meningite; a leucemia eosinofílica que foi afastada pelo hemograma e pela evolução.

O presente relato evidencia a necessidade de melhores métodos de pesquisa etiológica no exame de líquido céfalorraqueano nos casos de eosinofilorraquia.

\section{R E S M O}

E relatado o caso de um menino de 12 meses de idade que apresentava febre, rigidez de nuca e hepatoesplenomgalia e que, em dois exames sucessivos, apresentou eosinofilia sangüinea de $72 \%$ e $75 \%$ e liquórica de $75 \%$ e $78 \%$, respectivamente. A biópsia hepática mostrou um granuloma eosinofílico. Embora a criança apresentasse grande infestação por Ascaris lumbricoides, os autores admitem que o quadro clínico apresentado possa ter sido determinado por larvas de Toxocara canis, apesar do parasita não ter sido encontrado.

S U M M A R Y

Eosinophilic meningoencephalitis due to parasite associated with blood eosinophilia: a case report.

The case of a 12-month boy with hyperthermia, neck stifness, liver and spleen enlargement is reported. By two sucessive examinations there was found eosinophilia in the blood smear $(72 \%$ and $75 \%)$ and in the cerebrospinal fluid citology (75\% and $78 \%$ ). An eosinophilic granuloma was found by a liver biopsy. Although there was severe intestinal infestation by Ascaris lumbricoides, the authors believe that the clinical picture could be related to the Toxocara canis larvas (visceral larva migrans).

REFERENCIAS

1. ALLEMBY, C. F. \& SNELL, P. H. - Infection with adult Toxocara canis. Brit. Med. J. n. ${ }^{\circ}$ 5503: 1583, 1969.

2. ASCHKENASY, A. - Quelques données recentes sur le role physiologue des eosinophilies. Bull. Soc. Path. Exot. 55:499, 1962.

3. BAUER, J. - Diagnóstico Diferencial de las Enfermedades Internas. Segunda edição. Editorial Científico-Médica, Barcelona, 1959.

4. BEAVER, P. C.; SNYDER, C. H.; CARRERA, G. M.; DENT, J. H. \& LAFFERTY, J. W. - Chronic eosinophilia due to visceral larva migrans. Pediatrics 9:7, 1952.

5. BEAVER, P. C. - Toxocarosis (visceral larva migrans) in relation to tropical eosinophilia. Eull. Soc. Path. Exot. 55:555, 1962. 
6. BEAUTYMAN, W. \& WOOLF, A. L. - An ascaris larva in the brain in association with acute anterior poliomyelitis. J. Path. Bact. 63:635, 1951.

7. BLANC, F. \& NOSNY, Y. - Evolution de nos connaissances en matière d'eosinophilie tropicale et position actuelle du problème. Bull. Soc. Path. Exot. 55: 659,1962 .

8. BONNIN, H. \& MORETI, G. F. - Lois d'occurrence de l'eosinophilie dans les parasitoses animales. Prèsse Méd. 49:668, 1939.

9. BRAIN, R. \& ALLAN, B. - Encephalitis due to infection with Toxocara canis. Lancet n.o 7347: 1355, 1964.

10. BRUMPT, E.; AUDEBAUD, G.; KLEIN, J. M.; JOLLY, M.; MAZAUD, R. \& GOUBE, P. - Incidence d'Angiostrongylus cantonensis au Cambodge. Bull. Soc. Exot. 61:44, 1968.

11. CANELAS, H. M.; AIDAR, O. \& CAMPOS, E. P. - Esquistossomose com lesões meningo-radiculo-medulares. Arq. Neuro-Psiquiat. (São Paulo) 9:48, 1951.

12. CARVAlHO, O. \& SILVA, R. R. - Importância do leucócito eosinófilo em Pediatria. Med. Cirurg. Farm. (Rio de Janeiro) n.o 248:579, 1956.

13. CHIATANODH, H. \& ROSEN, L. - Fatal eosinophilic encephalomyelitis caused by the nematode Gnathostoma spinigerum. Am. J. Trop. Med. Hyg. 16:638, 1967.

14. CRISCI, A.; MOLESETTI, H. \& GHERARDI, J. — Eosinofilia en el líquido céfalorraquídeo. Rev. Tub. Uruguay 17:7, 1949.

15. COUTINHO, A. - Sindromes hipereosinofílicas pulmonares. Hospital (Rio de Janeiro) 72:77, 1967.

16. DESCHIENS, R. - Etude comparée des hypereosinophilies parasitaires et non parasitaires. Bull. Soc. Path. Exot. 55:598, 1962.

17. DESCHIENS, R. \& BENEX, J. - L'eosinophilie sanguine physiologique et juxtaphysiologique. Bull. Soc. Path. Exot. 55:529, 1962.

18. DODIN, A.; RATOVONDRAHEDY \& MOREAU, J. P. - Etude de l'eosinophilie dans la billarziose traitée (rectale et urinaire). Aperçu nouveau sur la pathogénie de l'eosinophilie. Bull. Soc. Path. Exot. 62:138, 1969.

19. DOUTHWAITE, A. H. — French's Index Differential Diagnosis. Oitava edięão. John Wright \& Sons Ltd., Bristol, 1960.

20. DUTREY, M. \& VELLUZ, J. - Hémorragie meningée d'origine vermineuse. Monde Méd. 49:668, 1939.

21. FERGUSON, R. L.; MC VAY, C. B. \& HILL, J. - Eosinophilic meningitis: two cases report. Am. J. Path. 26: 725, 1950.

22. GALLIARD, $\mathrm{H}$ - Le sindrome tropical de meningo-encephalite avec eosinophilie rachidienne. Bull. Soc. Path. Exot. 5:731, 1962.

23. GENTILINI, M. \& HAZARD, J. - Diagnostic d'une hyperéosinophilie sanguine. Prèsse Méd. 76:2279, 1968.

24. GRENET, P.; CHAVELET, F.; DUCAS, M. \& VILDE, J. J. - Les meningites aigues primitives à eosinophiles. Prèsse Méd. 75:1387, 1967.

25. HENAO, A. C. - Lesiones por Ascaris lumbricoides erraticos. Rev. Lat. Amer. Pat. 1:15, 1957.

26. HORIO, S. R. \& ALICATA, J. E. - Parasitic meningo-encephalitis in Havaii. A new parasitic disease of man. Havaii Med. J. 21:139, 1961.

27. JIMMEZ-QUIROS, O. - Ascariasis com manifestaciones nerviosas e eosinofilia del liquor. Rev. Biol. Trop. 7:67, 1959.

28. LAFON, R.; LABAUGE, R.; RIBSTEIN, M. \& BARJON, M. C. - Les eosinophilies du liquide céphalo-rachidien (La méningite aigue curable a eosinophilies). Rev. Neurol. (Paris) 97:466, 1957.

29. LANDSBOROUGH, D. - A case of cerebral paragonimiasis. J. Neurol. Neurocirur. Psychiat. 29:259, 1966.

30. LANGE, O. - Síndrome liquórica da cisticercose encefalo-meníngea. Rev. Neurol. Psiquiat. (São Paulo) 6:35, 1940.

31. MARAÑON, G. - Manual de Diagnóstico Etiológico. 10.a edição. Espasa-Calpe S.A., Madrid, 1957.

32. NIETO, D. \& ESCOBAR, I. A. - Estudio clínico e neuropatológico de los accidentes provocados por la vacinacion antirrabica. Bol. Inst. Estud. Med. Biol. $17: 27,1959$. 
33. NYE, S. W.; CHAPEL, H.; TANGCHAI, P.; SUNDARAKITI, S. \& PUNYAGUPTA, S. - Lesions of the brain in eosinophilic meningitis. Arch. Path. 89:9, 1970.

34. OH, J. S. - Paragonimus meningitis. J. Neurol. Sc. 6: 419, 1968.

35. PESSOA, S. B. - Parasitologia Médica. Quinta edição. Livraria Editora Guanabara, Rio de Janeiro, 1968.

36. PETTER, C. - Etude zoologique de la larva migrans. Ann. Parasitol. Hum. Comp. 35:118, 1960

37. PUNYAGUPTA, S.; LIMITRAKUL, C.; VICHIPANTHU, P.; KARNCHANACHETANEE, C. \& NYE, S. W. - Radiculomyeloencephalitis associated with eosinophilic pleocytosis. Am. J. Trop. Med. 17:551, 1960.

38. REIS, J. B. \& Bei, A. - O líquido céfalorraqueano no diagnóstico da criptococose do sistema nervoso. Arq. Neuro-Psiquiat. (São Paulo) 14:201, 1956.

39. ROSEN, L.; CHAPPELL, R.; LAQUEUR, G. L.; WALLACE, G. D.; WEINSTEIN, P. P. - Eosinophilic meningoencephalitis caused by a metastrongyloid lungworm of rats. J.A.M.A. 179:620, 1962.

40. SCHNEINER, J. - L'eosinophilie tropicale n'est pas une maladie. Bull. Soc. Path. Exot. 55:694, 1962.

41. SCHOLLHAMMER, G.; LEGAIT, J. P. \& COURDURIER, J. - Recherche sur Angiostrongylus cantonensis en République de Haute-Volta. Bull. Soc. Path. Exot. 61:441, 1968 .

42. SEPICH, M. J. \& MENDIZABAL, E. A. - Hidatidosis cerebral. J. Brasil. Neurol. 13:57, 1961 .

43. SHRAND, H. - Visceral larva migrans. Lancet n.o 7347: 1357, 1964.

44. SILVA, L. L. V. - Contribuição ao estudo de neuro-esquistossomose. Bol. Centro Estudos Hospital Servidores do Estado (Guanabara) 15:95, 1963.

45. SILVA, J. A. C. \& FLOSI, A. Z. - Sobre um caso de meningopatia secundária a ascaridíase. Rev. Neurol. Psichiat. de São Paulo 8:183, 1942.

46. SMIT, A M. - Eosinophilic meningitis at Kisaran (Indonesia) and the problem of its aetiology. Bull. Soc. Path. Exot. 55:722, 1962.

47. SNYDER, C. H. - Visceral larva migrans. Pediatrics 28:85, 1961

48. SOHIER, W. D. \& WILLIANS, R. A. - Myelofibrosis with anemia and eosinophilia. New England J. Med. 208:97, 1969.

49. SPINA-FRANCCA, A. - Aspectos biológicos de neuro-cisticercose: alterações do líquido céfalorraqueano. Arq. Neuro-Psiquiat. (São Paulo) 20:17, 1962.

50. SPINA-FRANCCA, A. - Síndrome liquórica da neurocisticercose. Arq. NeuroPsiquiat. (São Paulo) 19:307, 1961.

51. SPINA-FRANÇA, A. \& AMATO NETO, V. - o liquido céfalorraqueano na esquistossomose mansoni. Arq. Neuro-Psiquiat. (São Paulo) 13:274, 1955.

52. SUMMER, D. \& TINSLEY, E. G. F. - Encephalopathy due to visceral larva migrans. J. Neurol. Neurocirurg. Psichiat. 30:580, 1967.

53. VASQUEZ, L. E. - Meningitis con eosinofilia en el líquido céfalorraquideo seguida de un granuloma eosinofílico de la orbita. Arch. Col. Med. (El Salvador) $16: 29,1963$.

54. WEINGARTEN, R. J. — Tropical eosinophilia, Lancet 1:103, 1943.

55. WOODRUFF, A. W.; BISSERU, B. \& BOWE, J. C. - Infection with animal helmints as a factor in causing poliomyelitis and epilepsy. Brit. Med. J. 5503: 1576, 1966.

Hospital de Clínicas - Disciplina de Neurologia - 80000 Curitiba, PR - Brasil. 